\title{
Preparation and Evaluation of Solid Dispersions of Nitrendipine Prepared with Fine Silica Particles Using the Melt-Mixing Method
}

\author{
Liang WANG, ${ }^{a} \mathrm{Fu}$ De CuI, ${ }^{a}$ and Hisakazu SunADA ${ }^{*}, b$ \\ ${ }^{a}$ Department of Pharmaceutics, Shenyang Pharmaceutical University; No. 103, Wenhua Road, Shenyang 110016, P.R. \\ China: and ${ }^{b}$ Faculty of Pharmacy, Meijo University; 150 Yagatoyama, Tempaku-ku, Nagoya 468-8503, Japan. \\ Received June 23, 2005; accepted October 11, 2005
}

Solid dispersions (SD) of nitrendipine (NTD), a poorly water-soluble drug, were prepared using the meltmixing method with hydrophilic silica particles (Aerosil and Sylysia) with different particle size and specific surface areas as carriers. Powder X-ray diffraction and differential scanning calorimetry evaluation showed that NTD in the SDs treated with the melt-mixing method was dispersed in the amorphous state. FT-IR spectroscopy obtained with the SDs indicated the presence of hydrogen bonding between the secondary amine groups of NTD and silanol groups of silica particles. The dissolution property of NTD in the SDs was remarkably improved regardless of the grade of silica. At the end of the dissolution test $(60 \mathrm{~min})$ the concentrations of NTD for the SDs with Aerosil 200 and Sylysia 350 were 8.88 and $10.09 \mu \mathrm{g} / \mathrm{ml}$, corresponding to 28 and 31 times that of the original NTD crystals, respectively. The specific surface area and the adsorbed water amount of the SDs were also significantly improved. The rapid dissolution rate from the SDs was attributed to the amorphization of drug, improved specific surface area and wettability than the original drug crystals. In the stability test, powder X-ray diffraction pattern indicated that amorphous NTD in the SD with Aerosil 200 was stable for at least 1 month under the humid conditions $\left(40^{\circ} \mathrm{C} / 75 \% \mathrm{RH}\right)$.

Key words solid dispersion; silica particle; melt-mixing method; poorly water-soluble drug; nitrendipine

The solid dispersion method, by which a drug is dispersed in a carrier in the molecular state, is a useful pharmaceutical approach most commonly employed to improve the dissolution property and oral bioavailability of poorly water-soluble drugs. ${ }^{1-3)}$ Currently, various pharmaceutical approaches for the preparation of SDs, including coprecipitation, lyophilization, spray drying, solvent evaporation, fusion and cogrinding methods, have been reported. ${ }^{4-6)}$ However, most of these methods require a large amount of organic solvent to dissolve the drug and a water-soluble polymer. This causes environmental pollution and toxicity due to the residual solvent ${ }^{7)}$ and furthermore, in the common fusion method, tacky or glassy solids may cause undesirable processing problems. ${ }^{8)}$

Silica particles, characterized by the large specific surface area, are fine silicon dioxide particles with a high affinity with water molecules. The silanol groups on their surfaces can interact with the adsorbate through the hydrogen bonds. Products with various primary particle sizes and different BET-surface areas are available. Aerosil is non-porous colloidal silica with a nano-sized particle nature, and Sylysia is characterized by the large internal surface area with a large number of pores. The physicochemical properties of these two types of silica are shown in Table 1. Takeuchi et al. ${ }^{9-12)}$ have reported that SD particles with colloidal silica or porous silica prepared by the spray-drying method improve the dissolution properties of tolbutamide and indomethacin. Watanabe et al $^{13-15)}$ prepared SDs of indomethacin with silica nanoparticles using the co-grinding and melt-quenching method. A number of previous papers have reported that the drug molecules adsorbed onto the surface of the porous materials, ${ }^{16-18)}$ due to the mixing or heating method $^{19)}$ were found to exist in the amorphous state.

Nitrendipine (NTD) is a dihydropyridine calcium channel blocking agent ${ }^{20}$ producing peripheral vasodilation as its predominant in vivo effect with prolonged duration of action. ${ }^{21,22)}$ It has been extensively used to treat cardiovascular disorders ${ }^{23-25)}$ because of its greater selectivity for vascular conduction and contractility. ${ }^{26)}$ The molecular structure of NTD is illustrated in Fig. 1. Due to its low aqueous solubility (about $1.9-2.1 \mu \mathrm{g} / \mathrm{ml})^{27)}$ and high first-pass effect ${ }^{28,29)}$ leading to its poor absorption after oral administration, NTD often shows low and irregular bioavailability following oral administration. $^{30)}$ In the previous papers, sustained-release NTD microspheres with a solid dispersed structure have been prepared with Eudragit RS and Aerosil using a quasi-emulsion solvent diffusion method to improve the dissolution rate

Table 1. Physicochemical Properties of Aerosil and Sylysia of Various Grades

\begin{tabular}{cccccc}
\hline \hline Sample & $\begin{array}{c}\text { Hydrophilic } \\
\text { or } \\
\text { hydrophobic }\end{array}$ & $\begin{array}{c}\text { Porous } \\
\text { or } \\
\text { nonporous }\end{array}$ & $\begin{array}{c}\text { Particle } \\
\text { size } \\
(\mu \mathrm{m})\end{array}$ & $\begin{array}{c}\text { Specific } \\
\text { surface } \\
\text { area } \\
\left(\mathrm{m}^{2} / \mathrm{g}\right)\end{array}$ & $\begin{array}{c}\text { Pore } \\
\text { size } \\
(\mathrm{nm})\end{array}$ \\
\hline Aerosil 200 & & & 0.012 & 200 & - \\
Aerosil 300 & Hydrophilic & Nonporous & 0.007 & 300 & - \\
Aerosil 380 & & & 0.007 & 380 & - \\
Sylysia 350 & Hydrophilic & Porous & 3.9 & 300 & 21 \\
Sylysia 730 & Hydrop & 4.0 & 700 & 2.5 \\
\hline
\end{tabular}

The values were obtained from the catalogs of Aerosil (Nihon Aerosil) and Sylysia (Fuji Silysia).<smiles>COC(=O)C1=C(C)NC(C)=C(C(=O)OC)C1c1cccc([N+](=O)[O-])c1</smiles>

Fig. 1. Molecular Structure of Nitrendipine 


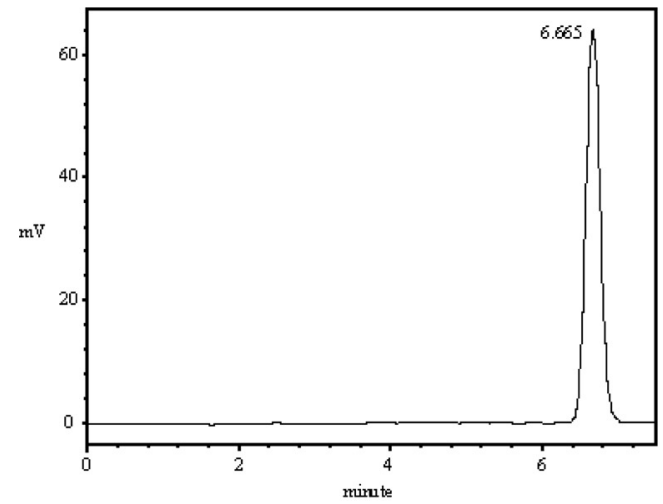

(A)

Fig. 2. HPLC Chromatograms of NTD before and after Being Heated

(A) before heated; (B) after heated (NTD-Aerosil 200 SD).

and bioavailability of NTD. ${ }^{31-34)}$ We also demonstrated that SDs of NTD prepared using the twin screw extruder method with HPMCP and Carbopol as carriers and the melt-mixing method with different grades of Aerosil improved the dissolution properties of NTD. ${ }^{35,36)}$

In the present study, we tried to prepare SD particles of NTD with porous silica (Sylysia 350, 730) and compared it to that with non-porous silica (Aerosil of different grades). The physicochemical properties of the SDs were investigated and compared with their physical mixtures.

\section{Experimental}

Materials Nitrendipine (NTD) was obtained from Nanjing Pharmaceutical Factory (China); as the carriers, fumed $\mathrm{SiO}_{2}$ powder (Aerosil 200, Aerosil 300 and Aerosil 380 from Nippon Aerosil Co., Japan) and porous silica (Sylysia 350 and Sylysia 730 from Fuji Silysia, Japan) were used. All other chemicals were of analytical grade or HPLC grade. All experiments were carried out under subdued light to prevent the degradation of NTD due to light.

Preparation of Solid Dispersion NTD and the two types of silica of different grades were mixed by hand in a polyethylene bag for $10 \mathrm{~min}$ with a weight ratio of $1: 5$ to obtain a physical mixture (PM), which was proved to have the highest dissolution than that of the weight ratio of $1: 1$ and $1: 3$ in our previous paper. ${ }^{36)}$ SD particles of NTD with fine silica particles were prepared using the melt-mixing method by heating the PMs at $180^{\circ} \mathrm{C}$, which was above the melting point of NTD, for $30 \mathrm{~min}$. The PMs and SDs were stored in a glass desiccator with blue silica gel for $1 \mathrm{~d}$ before testing their properties. The SDs prepared were analyzed with HPLC, and their chromatograms were found to be similar before and after being heated (Fig. 2).

Confirmation of Particle Morphology The morphology of the particles was observed with a scanning electron microscope (SEM, JEOL Type SM-T20).

Powder X-Ray Diffraction (PXRD) Powder X-ray diffraction analysis was performed with a linear X-ray diffraction system (RAD-2VC, Rigaku Denki Co., Ltd.) in which $\mathrm{CuK \alpha}$ rays $(40 \mathrm{kV}, 20 \mathrm{~mA})$ were used as X-rays. The degree of diffraction was measured at $5 \%$ min every $0.01^{\circ}$ between 5 and $45^{\circ}(2 \theta)$.

Differential Scanning Calorimetry (DSC) DSC analysis was carried out on a differential scanning calorimeter (DSC 60, Shimadzu Co., Japan). All samples were measured by placing $4-10 \mathrm{mg}$ of the powder into an aluminum pan for analysis. The thermograms were obtained by heating the samples at a rate of $10^{\circ} \mathrm{C} / \mathrm{min}$ from 30 to $180^{\circ} \mathrm{C}$ in atmospheric air. Plots of heat flow versus temperature were recorded.

Fourier Transformation IR Spectroscopy (FT-IR) Drug-carrier interactions in the SDs were determined based on IR spectra measured with a FTIR spectrometer (JASCO FT/IR-4100) by the $\mathrm{KBr}$ method in the 4000 $450 \mathrm{~cm}^{-1}$ region at a $4 \mathrm{~cm}^{-1}$ resolution at 64 scans per spectrum.

Specific Surface Area Measurement The specific surface area of the samples was measured with the argon (Ar) adsorption method using NOVA 1000 (Yuasa Ionics Inc.) after degassing the sample powders at $50{ }^{\circ} \mathrm{C}$ for

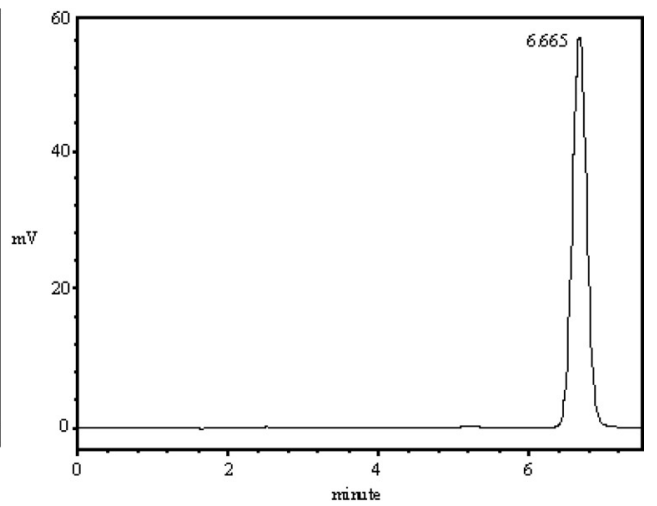

(B)

$30 \mathrm{~min}$.

Water Vapor Adsorption Analyses The amount of water adsorbed on the surface of the samples at different relative pressures was measured using a water vapor sorption analyzer (Hydrosorb 1000, Yuasa Ionics Inc.).

Dissolution Test The amount of drug released from the samples was determined using a dissolution test apparatus (NTR-3000, Toyama Sangyo Co., Ltd.) according to dissolution test method 2 (paddle method) described in the Japanese Pharmacopoeia (JP) XIX. The paddles were rotated at $100 \mathrm{rpm}$. The PMs and SDs equivalent to $40 \mathrm{mg}$ NTD were added to the dissolution medium $\left(900 \mathrm{ml}\right.$ water adjusted to $37 \pm 0.1^{\circ} \mathrm{C}$ ). Test fluids (about $5 \mathrm{ml}$ ) were withdrawn at predetermined time intervals from each vessel through a glass filter, and then filtered through a $0.2 \mu \mathrm{m}$ membrane filter (DISMIC-13CP, Toyo Filter Paper Co., Ltd.). After adding $1.4 \mathrm{ml}$ methanol to $0.6 \mathrm{ml}$ filtrate, the concentration of NTD was measured using a Shodex HPLC system (Showa Denko, Tokyo, Japan). The same volume of fresh medium was replaced and the correction for the cumulative dilution was calculated. The amount of NTD dissolved for each sample $(n=3)$ was plotted versus time. The HPLC conditions were as follows: reverse-phase column, Inertsil ODS-3 ( $5 \mu \mathrm{m}, 46 \mathrm{~mm} \phi \times 150 \mathrm{~mm}$, GL Science Inc., Tokyo, Japan) at $35^{\circ} \mathrm{C}$; mobile phase, methanol: water $=7: 3(\mathrm{v} / \mathrm{v})$ mixture; flow rate, $1.0 \mathrm{ml} / \mathrm{min}$; detection wavelength, $236 \mathrm{~nm}$.

Stability Test The sample particles were stored in the desiccators with $75 \% \mathrm{RH}$ (saturated solution with $\mathrm{NaCl}$ ) at $40^{\circ} \mathrm{C}$. The crystallinity of NTD in the SD particles was measured with the powder X-ray diffraction method.

\section{Results and Discussion}

Confirmation of Particle Morphology The SEM photographs of NTD-Aerosil 200 systems and NTD-Sylysia 350 systems are shown in Fig. 3. The crystal of NTD was observed in Fig. 3A. It was confirmed from Fig. 3B that the Aerosil 200 particles formed agglomerates. In NTD-Aerosil 200 PM (Fig. 3C) and NTD-Sylysia 350 PM (Fig. 3F), residual crystals of NTD were observed between the silica particles, which were not observed for the SDs (Figs. 3D, G). This suggested that the drug was well dispersed on the surface of silica particles in the SDs.

Changes in Crystallinity Powder X-ray diffraction patterns of NTD-Aerosil (1:5) systems and NTD-Sylysia $(1: 5)$ systems prepared by the melt-mixing method are shown in Fig. 4. Pure NTD drug powder showed numerous distinctive peaks (Fig. 4A) that indicated a high crystallinity. In the NTD-Aerosil PMs (Figs. 4B, D, F) and NTD-Sylysia PMs (Figs. 4H, J), the distinctive diffraction peaks of NTD persisted with a marked decrease in their intensity compared with original NTD crystals. Both the NTD-Aerosil SDs (Figs. 4C, E, G) and the NTD-Sylysia SDs (Figs. 4I, K) treated with the melt-mixing method exhibited a completely 
halo pattern of the SD with no diffraction peaks derived from NTD observed. This indicated that NTD in the SDs was transformed to an amorphous state with the melt-mixing method.
(A)

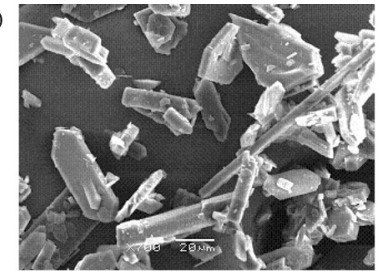

(C)

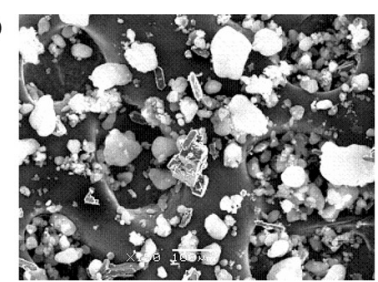

(E)

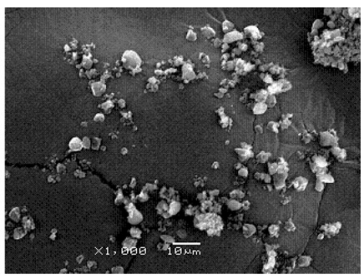

(G)

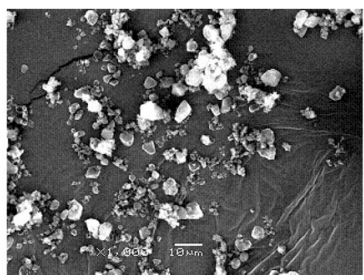

(B)

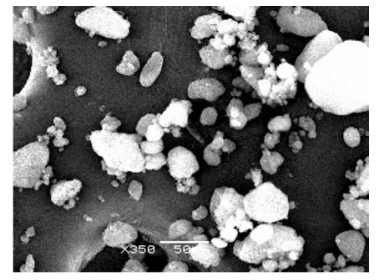

(D)

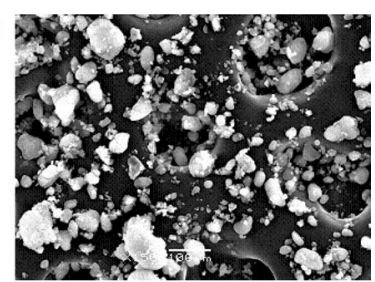

(F)

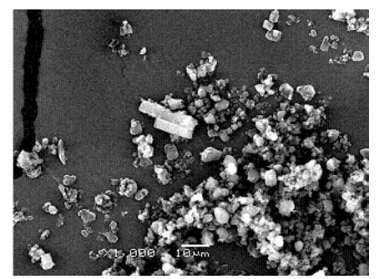

Fig. 3. Scanning Electron Micrographs of NTD-Aerosil 200 Systems and NTD-Sylysia 350 Systems

(A) NTD; (B) Aerosil 200; (C) PM with Aerosil 200; (D) SD with Aerosil 200; (E) Sylysia 350; (F) PM with Sylysia 350; (G) SD with Sylysia 350.
DSC curves of NTD in SD particles are exhibited in Fig. 5. Table 2 summarizes the onset melting points and fusion enthalpy $(\Delta H)$ of NTD-silica systems. The degree of crystallinity $\left(X_{\mathrm{cr}}\right)$ of samples was calculated according to Eq. 1:

$$
X_{\text {cr }}=\left(\Delta H / \Delta H_{0}\right) \times 100 \%
$$

where $\Delta H$ is the fusion enthalpy of NTD in the samples and $\Delta H_{0}$ is the fusion enthalpy of the original NTD crystalline structure. The DSC thermogram of pure NTD (Fig. 5A) showed a sharp endothermic peak with an onset melting temperature of $157.7^{\circ} \mathrm{C}$ and a fusion enthalpy $(\Delta H)$ of about $94.66 \mathrm{~J} / \mathrm{g}$. Endothermic peaks corresponding to pure NTD were observed with the NTD-Aerosil PMs (Figs. 5B, D, F) and NTD-Sylysia PMs (Figs. 5H, J). Furthermore, as evident from Table 2, $\Delta H$ and the crystallinity of the PMs markedly decreased and the onset melting points of the PMs, containing whether Aerosil 200, 300, 380 or Sylysia 350, 730, shifted toward a lower temperature. This phenomenon that the drug melting point decreases after being adsorbed on the porous materials has been reported ${ }^{17,18)}$ and indicated the existence of some interaction between NTD and the silica particles in the PMs. NTD-Aerosil SDs (Figs. 5C, E, G) and NTD-Sylysia SDs (Figs. 5I, K) prepared by the melt-mixing method showed broad curves with no endothermic peak corresponding to the melting of pure crystalline NTD. This observation confirmed that NTD changed to an amorphous state and the SDs were formed when treated with the meltmixing method.

Confirmation of Interactions by IR Spectrum It is known that the major adsorption sites on a silica surface are surface hydroxyl groups. The interaction between drug molecules and silanol groups of silica has been reported previously. ${ }^{12-15)}$ In order to test for possible intermolecular interactions between NTD and silica particles, FT-IR was used. FT-IR spectra obtained with samples prepared using the melt-mixing method with Aerosil as the carrier are shown in Fig. 6, and those obtained with NTD-Sylysia systems are shown in Fig. 7. The wave numbers of the characteristic peaks are summarized in Table 3. NTD alone (Fig. 6A),
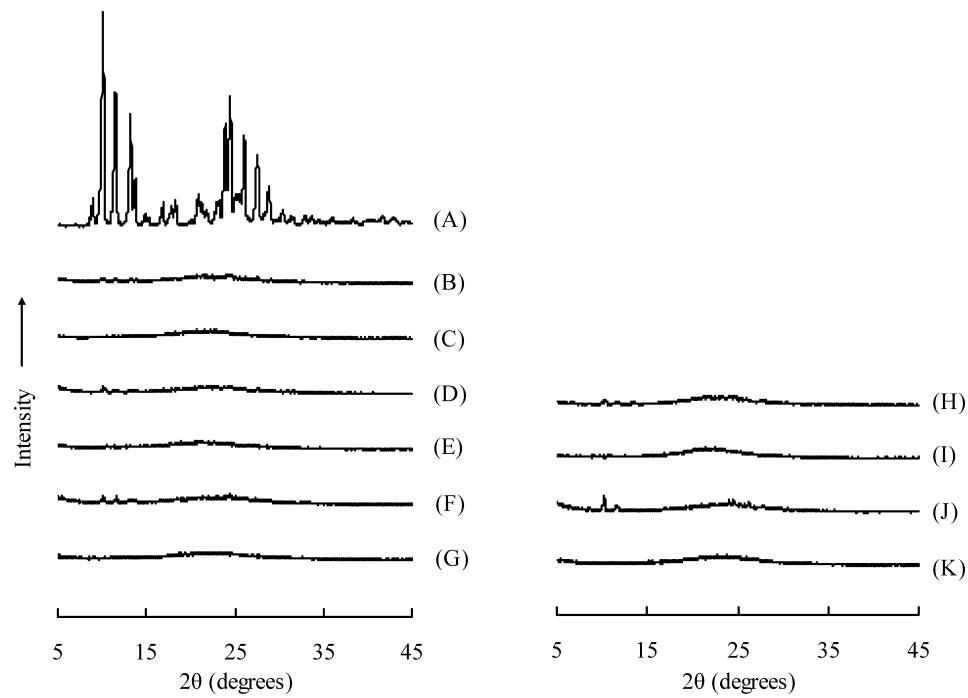

Fig. 4. Powder X-Ray Diffraction Patterns of NTD-Aerosil Systems and NTD-Sylysia Systems Prepared with the Melt-Mixing Method

(A) NTD; (B) PM with Aerosil 200; (C) SD with Aerosil 200; (D) PM with Aerosil 300; (E) SD with Aerosil 300; (F) PM with Aerosil 380; (G) SD with Aerosil 380; (H) PM with Sylysia 350; (I) SD with Sylysia 350; (J) PM with Sylysia 730; (K) SD with Sylysia 730. 


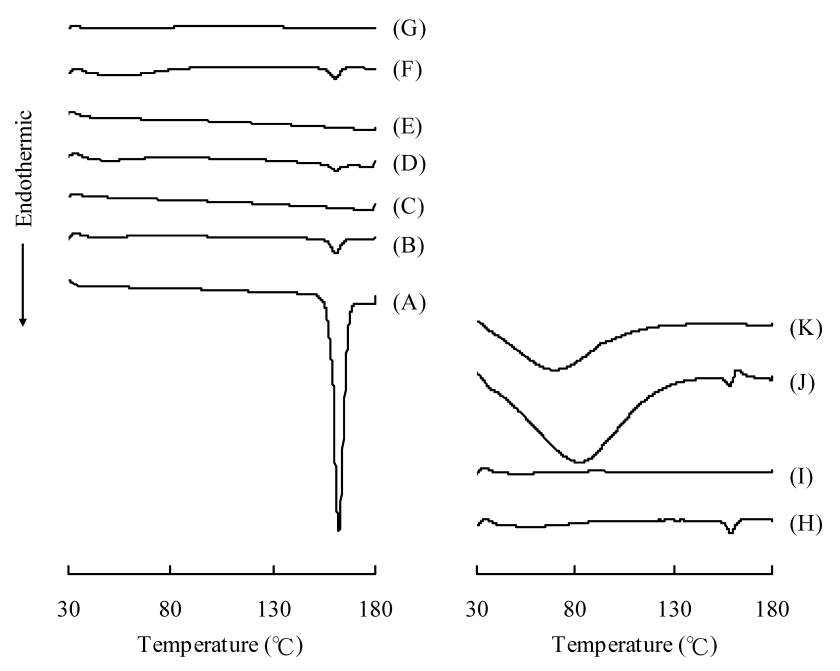

Fig. 5. Differential Scanning Calorimetry Curves of NTD-Aerosil Systems and NTD-Sylysia Systems Prepared with the Melt-Mixing Method

(A) NTD; (B) PM with Aerosil 200; (C) SD with Aerosil 200; (D) PM with Aerosil 300; (E) SD with Aerosil 300; (F) PM with Aerosil 380; (G) SD with Aerosil 380; (H) PM with Sylysia 350; (I) SD with Sylysia 350; (J) PM with Sylysia 730; (K) SD with Sylysia 730 .

Table 2. Fusion Enthalpy and Onset Melting Temperature of NTDAerosil Systems and NTD-Sylysia Systems

\begin{tabular}{lccc}
\hline \hline \multicolumn{1}{c}{ Sample } & $\begin{array}{c}\text { Melting point } \\
\left({ }^{\circ} \mathrm{C}\right)\end{array}$ & $\begin{array}{c}\text { Fusion enthalpy } \\
(\mathrm{J} / \mathrm{g})\end{array}$ & $\begin{array}{c}X_{\mathrm{cr}} \\
(\%)\end{array}$ \\
\hline NTD & 157.73 & 94.66 & 100 \\
NTD-Aerosil 200 PM & 156.3 & 12.8 & 13.52 \\
NTD-Aerosil 300 PM & 156.38 & 5.89 & 6.22 \\
NTD-Aerosil 380 PM & 155.92 & 7.96 & 8.41 \\
NTD-Sylysia 350 PM & 154.7 & 9.11 & 9.62 \\
NTD-Sylysia 730 PM & 155.16 & 3.91 & 4.13 \\
NTD-Aerosil 200 SD & - & - & - \\
NTD-Aerosil 300 SD & - & - & - \\
NTD-Aerosil 380 SD & - & - & - \\
NTD-Sylysia 350 SD & - & - & - \\
NTD-Sylysia 730 SD & - & - & - \\
\hline
\end{tabular}

-, endothermic peaks were not detected.

NTD-Aerosil 200 PM (Fig. 6C) and NTD-Sylysia 350 PM (Fig. 7C) showed N-H stretching vibrations due to the secondary amine groups (-NH-) of NTD at 3316 and 3319.86 $\mathrm{cm}^{-1}$, respectively, which was not observed for NTD-Sylysia 730 PM. The N-H stretching vibrations disappeared in the FT-IR spectra of NTD-Aerosil SDs (Figs. 6D, E, F) and NTD-Sylysia SDs (Figs. 7D, F).

The characteristic absorption peaks due to the silanol groups made red shifts in the case of all the PMs, while the blue shifts of silanol groups to lower wave numbers were observed for all the SDs, according to Table 3. These findings suggested the hydrogen bonding between the secondary amine groups of NTD and silanol groups of silica formed during the formation of SDs using the melt-mixing method.

Specific Surface Area The specific surface areas of the samples measured using the argon adsorption method are shown in Table 4. There was an appreciable increase in the specific surface area of the SDs compared with that of pure NTD $\left(0.49 \mathrm{~m}^{2} / \mathrm{g}\right)$. However, the specific surface area of SDs was found to cause a decrease compared with that of the corresponding PMs. These findings are consistent with the ob-

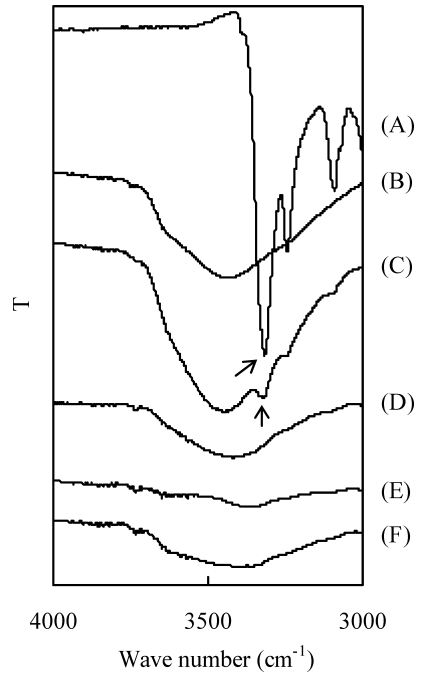

Fig. 6. FT-IR Spectra of NTD-Aerosil Systems

(A) NTD; (B) Aerosil 200; (C) PM with Aerosil 200; (D) SD with Aerosil 200; (E) SD with Aerosil 300; (F) SD with Aerosil 380.

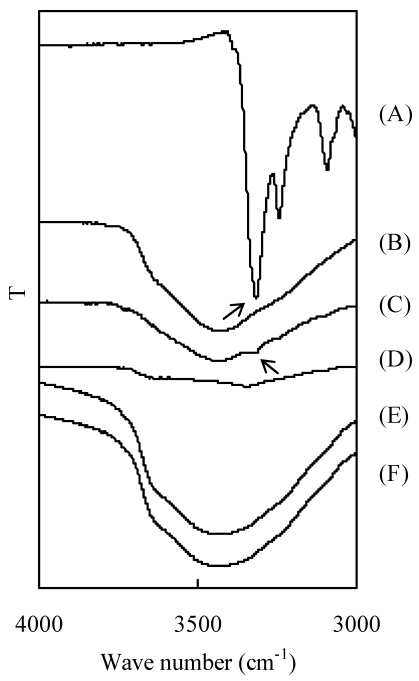

Fig. 7. FT-IR Spectra of NTD-Sylysia Systems

(A) NTD; (B) Sylysia 350; (C) PM with Sylysia 350; (D) SD with Sylysia 350; (E) PM with Sylysia 730; (F), SD with Sylysia 730.

Table 3. Summarization of Wavenumber of the Characteristic Peaks of NTD-Aerosil Systems and NTD-Sylysia Systems

\begin{tabular}{lcc}
\hline \multicolumn{1}{c}{ Sample } & Silanol group & - NH- group \\
\hline \hline NTD & - & 3316 \\
Aerosil 200 & 3434.60 & - \\
Sylysia 350 & 3433.60 & - \\
NTD-Aerosil 200 PM & 3444.24 & 3319.86 \\
NTD-Aerosil 200 SD & 3420.14 & - \\
NTD-Aerosil 300 SD & 3358.43 & - \\
NTD-Aerosil 380 SD & 3420.14 & - \\
NTD-Sylysia 350 PM & 3443.28 & - \\
NTD-Sylysia 350 SD & 3345.89 & - \\
NTD-Sylysia 730 PM & 3465.46 & - \\
NTD-Sylysia 730 SD & 3433.64 & \\
\end{tabular}

servations in the previous studies. ${ }^{10,17,19)}$ It was concluded that the drug particles were made uniformly dispersed and tended to form hydrogen bonding with the silanol groups on 
Table 4. Specific Surface Area of NTD-Aerosil Systems and NTDSylysia Systems

\begin{tabular}{cll}
\hline \hline Carriers & PM & SD \\
\hline Aerosil 200 & 117.71 & 108.08 \\
Aerosil 300 & 196.05 & 137.9 \\
Aerosil 380 & 207.22 & 146.06 \\
Sylysia 350 & 171.37 & 151.86 \\
Sylysia 730 & 548.39 & 144.56 \\
\hline
\end{tabular}

the surface of the silica particles due to the melt-mixing effect during the formation of SDs, and the adsorption of NTD onto the surface of Aerosil or into the pores of Sylysia led to a decrease of the specific surface area. Especially in porous Sylysia 730 with a thinner pore size $(2.5 \mathrm{~nm})$ than Sylysia $350(21 \mathrm{~nm})$, the pores might be covered by the drug molecules which led to the diminution of the specific surface area.

Water Vapor Adsorption Analyses Water adsorption has been used to characterize the particle wettability of nonporous colloidal silica. ${ }^{37)}$ In particular, water adsorption kinetics at saturated vapor pressure was correlated with advancing water contact angles and the surface coverage of hydroxyl groups, thus enabling the concentration and reactivity of surface hydroxyl groups to be estimated. A good correlation was observed between the hydroxylation-state of silica and the contact angle, concluding that there is an interplay between the surface chemistry, water adsorption and particle wettability. Hence, the wettability of SD particles prepared by the melt-mixing method was investigated with the water vapor adsorption analyses.

The water vapor adsorption isotherms of NTD-Aerosil (1:5) systems and NTD-Sylysia (1:5) Systems are shown in Figs. 8 and 9, respectively. The amount of water adsorbed on the surface of pure NTD, Aerosil 200 and Sylysia 350 at 0.4 relative pressure was $0.28,11.78$ and $26.90 \mathrm{ml} / \mathrm{g}$ respectively, indicating the poor wettability of NTD and the hydrophilicity of silica particles. In the case of NTD-Aerosil systems (Fig. 8) and the NTD-Sylysia 350 system (Fig. 9), the isotherm assumed a convex (to the relative pressure axis) appearance, which gave a strong indication of no monolayer formation plus a delayed multilayer. Differently, the NTD-Sylysia 730 system showed a sigmoidal isotherm typical of hydrophilic surfaces demonstrating both monolayer and multilayer formation.

All the SDs were found to produce a significant increase in the amount of water adsorbed over pure NTD, suggesting the improvement of the wettability of the SD particles. The amount of water adsorbed on the surface of the SDs at 0.4 relative pressure (Table 5) accords to the following order: Sylysia 730 $>$ Sylysia 350 $>$ Aerosil 380 $>$ Aerosil 300 $>$ Aerosil 200. It was concluded that there would be more silanol groups on the surface of silica particles with larger specific surface areas to increase the amount of adsorbed water. However, a slight decrease in the amount of water adsorbed of the SDs compared with the PMs was observed. It was considered that the adsorption of drug molecules on the surface or in the pores of silica particles through the hydrogen bonding with silanol groups decreased the surface coverage of silanol groups, which leads to the decrease of the adsorbed water amount of the SDs.

Dissolution Test Dissolution profiles of NTD from

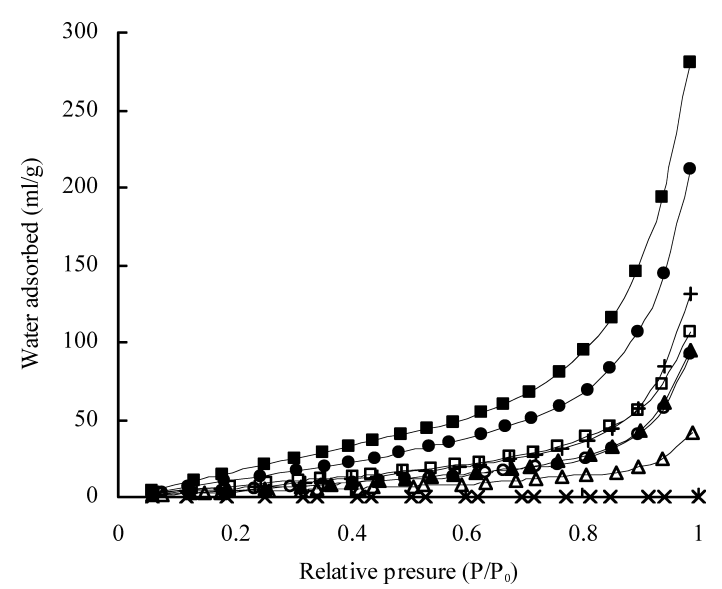

Fig. 8. Water Vapor Adsorption Isotherm of NTD-Aerosil Systems

$\times$, NTD; +, Aerosil 200; $\triangle$, $\boldsymbol{\Delta}$, Aerosil 200; ○, ९, Aerosil 300; $\square, \mathbf{\square}$, Aerosil 380; open symbols, solid dispersions; closed symbols, physical mixtures.

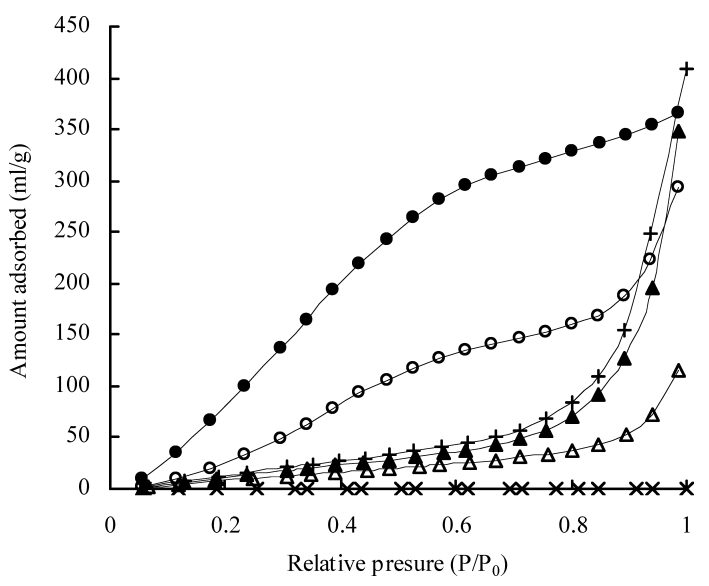

Fig. 9. Water Vapor Adsorption Isotherm of NTD-Sylysia Systems $\times$, NTD; +, Sylysia 350; $\triangle$, $\boldsymbol{\Delta}$, Sylysia 350; $\bigcirc, \bullet$, Sylysia 730; open symbols, solid dispersions; closed symbols, physical mixtures.

Table 5. Amount of Water Adsorbed on the Surface of NTD-Aerosil Systems and NTD-Sylysia Systems at 0.4 Relative Pressure

\begin{tabular}{ccc}
\hline \hline Carriers & PM & SD \\
\hline Aerosil 200 & 8.67 & 5.57 \\
Aerosil 300 & 22.27 & 8.96 \\
Aerosil 380 & 32.6 & 13.54 \\
Sylysia 350 & 22.74 & 15.32 \\
Sylysia 730 & 192.84 & 78.44 \\
\hline
\end{tabular}

NTD-Aerosil systems and NTD-Sylysia systems are presented in Figs. 10 and 11, respectively. For pure NTD crystals, at the end of dissolution test $(60 \mathrm{~min})$ the concentration of NTD was only $0.32 \mu \mathrm{g} / \mathrm{ml}$, indicating the very poor dissolution property of the drug. Dissolution of NTD from all the SDs, whether prepared with non-porous Aerosil or with porous Sylysia, was significantly higher than from pure NTD and PMs. Moreover, both NTD-Aerosil SDs and NTDSylysia SDs provided a higher supersaturation level of the drug in the dissolution, indicating that NTD in SDs with silica particles prepared with the melt-mixing method exists in an amorphous form, thereby inducing not only a size reduction of the drug but also the good wettability of the drug. The 


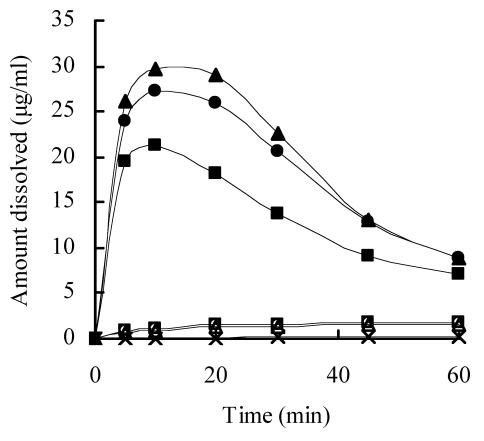

Fig. 10. Dissolution Profiles of NTD from NTD-Aerosil Systems Prepared with the Melt-Mixing Method Using Aerosil of Different Grades

$\times$, NTD; $\triangle, \boldsymbol{\Delta}$, Aerosil 200; $\bigcirc, \boldsymbol{\bullet}$, Aerosil 300; $\square, \boldsymbol{\square}$, Aerosil 380; open symbols, physical mixtures; closed symbols, solid dispersions. Each point represents the mean $(n=3)$.

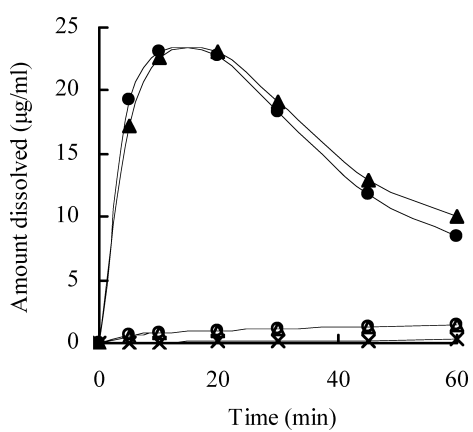

Fig. 11. Dissolution Profiles of NTD from NTD-Sylysia Systems Prepared with the Melt-Mixing Method Using Sylysia of Different Grades

$\times$, NTD; $\triangle, \boldsymbol{\Delta}$, Sylysia 350 ; $\bigcirc, \bullet$, Sylysia 730 ; open symbols, physical mixtures; closed symbols, solid dispersions. Each point represents the mean $(n=3)$.

concentrations of NTD after $60 \mathrm{~min}$ for SDs with Aerosil 200, Aerosil 300 and Aerosil 380 were 8.88, 8.81 and $7.00 \mu \mathrm{g} / \mathrm{ml}$, corresponding to a $28-, 27-$ and 22 -fold increase in the intrinsic drug dissolution, and for SDs with Sylysia 350 and Sylysia 730, they were 10.09 and $8.43 \mu \mathrm{g} / \mathrm{ml}$, corresponding to 31 and 26 times that of original NTD crystals, respectively.

The rank order of the drug supersaturation concentration at about $10 \mathrm{~min}$ for SDs was Aerosil $200(29.78 \mu \mathrm{g} / \mathrm{ml})>$ Aerosil $300(27.23 \mu \mathrm{g} / \mathrm{ml})>$ Sylysia $730 \quad(23.11 \mu \mathrm{g} / \mathrm{ml})>$ Sylysia $350(23.04 \mu \mathrm{g} / \mathrm{ml})>$ Aerosil $380(21.37 \mu \mathrm{g} / \mathrm{ml})$. It can be presumed that the dissolution rate of the drug from the surface of SDs with non-porous silica particles is higher than that from the pores of porous silica particles.

Moreover, the particles of pure NTD and PMs were found to be floating on the surface of the dissolution medium during the dissolution experiment. Conversely, the SDs were well dispersed as soon as they were added to the dissolution medium. This result implied that the hydrophilic properties of the silica particles contributed to the improved wettability and improved drug dissolution from the SD.

Accordingly, both the amorphization of the drug and the good wettability of SDs might account for the faster dissolution rate of SDs compared with PMs.

Stability Test Figure 12 shows the stability of amorphous NTD in the SD with Aerosil 200 stored at $75 \% \mathrm{RH}$ and $40{ }^{\circ} \mathrm{C}$. It was found that the amorphous state of NTD in NTD-Aerosil 200 SD particles was well maintained under moist conditions within one month. This may be attributed to

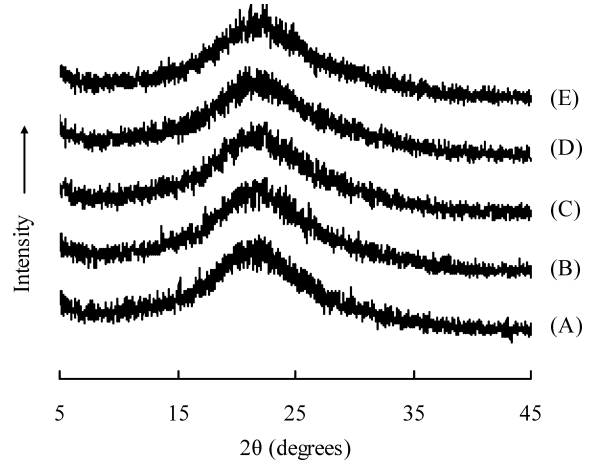

Fig. 12. Stability of Amorphous NTD in Solid Dispersion Particles with Aerosil 200 Stored at $75 \% \mathrm{RH}$ and $40{ }^{\circ} \mathrm{C}$

(A) $0 \mathrm{~d}$; (B) $1 \mathrm{~d}$; (C); 1 week; (D) 2 weeks; (E) 1 month.

the hydrogen bonds between the secondary amine groups of NTD and the silanol groups of silica in the SDs prepared using the melt-mixing method, which inhibited the crystallization of the drug from the amorphous state. Takeuchi et $a l .{ }^{11)}$ reported that Sylysia 350 had a strong stabilizing effect on the meta-stable tolbutamide in SD particles compared with Aerosil 200, probably because of their porous structure. In the present case, it was considered that non-porous Aerosil 200 of nano-sized particles also had a stabilizing effect on the amorphous NTD in the SDs prepared using the melt-mixing method.

\section{Conclusions}

SDs of NTD were prepared with the melt-mixing method using silica particles as carriers. NTD was found to be transformed to an amorphous state in SDs with the melt-mixing method. The dissolution property, specific surface area and wettability of NTD were improved. For NTD-Aerosil 200 $\mathrm{SD}$, the dissolution rate was faster than that of NTD-Sylysia SDs, while the specific surface area and the adsorbed water amount were less than that of NTD-Sylysia systems. This is considered to be due to the pores of Sylysia, as mentioned above. The interaction of hydrogen bonding between NTD and silanol groups of silica particles was confirmed by its IR spectrum. The melt-mixing method is considered to be a useful method for preparing SDs with silica particles.

It was observed that the high supersaturation concentration of the drug after about 10 min was not maintained continuously during the dissolution test of the various SDs. Hence it was necessary to maintain the supersaturation of the drug using high-molecular-weight polymer or other new methods to improve the dissolution properties of NTD further.

\section{References}

1) Sekiguchi K., Obi N., Chem. Pharm. Bull., 9, 866-872 (1961).

2) Chiou W. L., Riegelman S., J. Pharm. Sci., 59, 937-942 (1970).

3) Chiou W. L., Riegelman S., J. Pharm. Sci., 60, 1281-1302 (1971).

4) Shakhtshneider T. P., Vasiltchenko M. A., Politov A. A., Boldyrev V. V., Int. J. Pharm., 130, 25-32 (1996).

5) Sudha R. V., Karin A. M., Siva T., David J. W. G., Int. J. Pharm., 236, $111-123$ (2002).

6) Van den Mooter G., Augustijns P., Blaton N., Kinget R., Int. J. Pharm., 164, 67-80 (1998).

7) Ford J. L., Stewart A. F., Rubinstein M. K., J. Pharm. Pharmacol., 31, $726-729$ (1979).

8) Summers M. P., Enever R. P., J. Pharm. Sci., 65, 1613-1617 (1976).

9) Takeuchi H., Handa T., Kawashima Y., Chem. Pharm. Bull., 35, 3800-3806 (1987). 
10) Takeuchi H., Nagira S., Yamamoto H., Kawashima Y., J. Soc. Powder Technol., Jpn., 40, 157-162 (2003).

11) Takeuchi H., Nagira S., Yamamoto H., Kawashima Y., Powder Technol., 141, 187-195 (2004).

12) Takeuchi H., Nagira S., Yamamoto H., Kawashima Y., Int. J. Pharm., 288, 177-183 (2005).

13) Watanabe T., Wakiyama N., Usui F., Ikeda M., Isobe T., Senna M., Int. J. Pharm., 226, 81-91 (2001).

14) Watanabe T., Ohno I., Wakiyama N., Kusai A., Senna M., S.T.P. Pharm. Sci., 12, 363-367 (2002).

15) Watanabe T., Hasegawa S., Wakiyama N., Kusai A., Senna M., Int. J. Pharm., 250, 283-286 (2003).

16) Matsumoto K., Nakai Y., Yonemochi E., Oguchi T., Yamamoto K., Int. J. Pharm., 108, 167-172 (1994).

17) Nakai Y., Yamamoto K., Terada K., Ichikawa J., Chem. Pharm. Bull., 32, 4566-4571 (1984).

18) Matsumoto K., Nakai Y., Yonemochi E., Oguchi T., Yamamoto K., Chem. Pharm. Bull., 46, 314-318 (1998).

19) Kinoshita M., Baba K., Nagayasu A., Yamabe K., Shimooka T., Takeuchi Y., Azuma M., Houchi H., Minakuchi K., J. Pharm. Sci., 91, $362-370$ (2002).

20) Mikus G., Eichelbaum M., J. Cardiovasc. Pharmacol., 9, 140-141 (1987).

21) Knorr K., Stoepel K., Arzneim.-Forsch., 31, 2062 -2064 (1981).

22) Muller F., Bolli P., Erne P., Block L., Kiowsky W., Buhler F., J. Cardiovasc. Pharmacol., 6, 173-176 (1984).

23) Stoepel K., Heise A., Kazda S., Arzneim.-Forsch., 31, 2056-2061
(1981).

24) Santiago T. M., Lopez L. M., Ann. Pharmacother, 24, 167-175 (1990).

25) Hasegawa G. R., Clin. Pharm., 7, 97-108 (1988).

26) Schneeweiss A., Pediatr. Cardiol., 9, 109-115 (1988).

27) Kristl A., Primoič S., Strojan P., Kozjek F., Pharmazie, 47, 704-706 (1992).

28) Krol G. J., Lettieri J. T., Yeh S. C., Burkholder D. E., Birkett J. P., J. Cardiovasc. Pharmacol., 9, 122-128 (1987).

29) Goda K. L., Sorkin E. M., Drugs, 33, 123-155 (1987).

30) Zhu Z., Mao F., Zhu J., Yao Xue Xue Bao, 25, 709_716 (1990).

31) Cui F., Yang M., Jiang Y., Cun D., Lin W., Fan Y., Kawashima Y., J. Control Release, 91, 375-384 (2003).

32) Yang M., Cui F., You B., Fan Y., Wang L., Yue P., Yang H., Int. J. Pharm., 259, 103-113 (2003).

33) Yang M., Cui F., You B., You J., Wang L., Zhang L, Kawashima Y., J. Control Release, 98, 219-229 (2004).

34) Yang M., Cui F., You B., Wang L., Yue P., Kawashima Y., Int. J. Pharm., 286, 99-109 (2004).

35) Wang L., Cui F., Hayase T., Sunada H., Chem. Pharm. Bull., 53, $1240-1245$ (2005).

36) Wang L., Sunada H., "Preparation and evaluation of solid dispersion for nitrendipine-Aerosil system using melt-mixing method," Symposia of the 125th Annual Meeting of the Pharmaceutical Society of Japan, 2005.

37) Tim H. M., Clive A. P., Robert A. H., Colloids Surf. A, 176, 253-266 (2001). 